\title{
THE CCD/TRANSIT INSTRUMENT (CTI) BLUE OBJECT SURVEY
}

\author{
J. Davy Kirkpatrick and John T. McGraw \\ Steward Observatory, University of Arizona \\ Tucson, Arizona 85721, U.S.A.
}

\section{Introduction:}

The CCD/Transit Instrument (CTI) is a $1.8 \mathrm{~m}, \mathrm{f} / 2.2$ meridian-pointing telescope located on Kitt Peak. It has no moving parts, but utilizes two RCA charge-coupled devices (CCDs) aligned with columns in the east-west direction and operated in the "time-delay and integrate" (TDI) mode at the apparent sidereal rate to form an image of the sky as it transits (McGraw et al. 1986). The strip is 8.25 arcminutes wide north-south with length determined by the length of the night. Thus, each night the CTI surveys about 15 square degrees of the sky, or about 45 square degrees per year, to a nightly limiting magnitude of $\mathrm{mv} \geq 20$. One of the two CCDs in the focal plane always observes through a $V$ filter while the other utilizes one of $(U), B, R$, or $I$, depending upon the sky brightness. These data are searched to find every detectable object and photometric parameters are calculated for each. These data become part of a Master List which contains the best estimate for each parameter and a History List which maintains a light curve for every detected object. The vital statistics of the project are listed in Table 1.

This paper describes our preliminary efforts at investigating the blue stellar component of these data bases. The CTI survey yields high-precision, homogeneous multi-color photometry to faint limiting magnitudes, thus allowing a statistically complete sample of objects which can be used for a wide variety of scientific programs. The photometric precision derives principally from utilization of the TDI technique which effectively averages all spatial instrumental corrections (bias and flat-field) into more stable linear functions.

In particular, we wish to spectroscopically identify white dwarf and subdwarf stars to faint limiting magnitude both in the galactic halo and in the disk. These stars can be used as probes of galactic structure in the solar vicinity. Thus far we have spectroscopically identified 110 objects which have been determined to be "very blue" on the basis of colors derived from B, V, R and I measurements. We use the spectroscopic identifications to refine the loci of white dwarfs, subdwarfs, etc. in our $\mathrm{N}$-dimensional color space to fainter limiting magnitudes. Additionally, we begin an investigation of the motions and velocities of the white dwarfs we have discovered.

\section{The Survey:}

The blue candidates were selected from their outlying positions in color-magnitude or colorcolor diagrams. Each blue candidate is visually inspected in the CTI pixel data to ensure it is not contaminated by the halo of a nearby very bright star or merged with one or more stars. We also 
reject candidates contaminated by CCD artifacts such as charge "bleeding" from bright stars. The objects which survive our visual test are promoted to our observing list.

\section{TABLE 1: Survey Vital Statistics}

$1.8 \mathrm{~m}, \mathrm{f} / 2.2$ Automated Telescope

Two RCA CCDs - $320 \times 512$ Pixels, 30 Microns in Size

Field Scale of 52 Arcsec $\mathrm{mm}^{-1}$ - Pixel Subtends 1.55 Arcsec

Declination of Strip - +28 Degrees

Width of Surveyed Strip - 8.26 Arcmin

Total Area Surveyed - 43.7 Square Degrees

Time History Recorded in V Bandpass

Time-averaged (U), B, V, R, I Colors Obtained

\section{DETECTORS:}

CCDs Have No Cosmetic Blemishes

"V" CCD - 40 Electron Readout Noise

- 10.78 Electrons/ADU

- Nightly Limiting Magnitude $\mathrm{V}=21$

"Color" CCD - 72 Electron Readout Noise

- 9.18 Electrons/ADU

\section{CONTROL AND ACQUISITION COMPUTER SYSTEM: \\ - 16-bit Minicomputer \\ - Real-time Program "Herschel" Requires Minimal Human Response \\ - Data Stored on Disk During Acquisition \\ - Data Written to Magnetic Tape for Transport to Tucson}

Thus far we have had 1.5 clear nights of spectroscopic observing time on the $4.5 \mathrm{~m}$ Multiple Mirror Telescope (MMT) and 6.5 nights on Steward Observatory's $2.3 \mathrm{~m}$ telescope on Kitt Peak. Preliminary statistical results are presented in Table 2 . At the $2.3 \mathrm{~m}$, we identify those objects with $m_{V} \leq 18.0$ in about 60 minutes; at the MMT we easily reach $m_{V}=19.5$ in the same amount of time. Because the CTI photometric system must be "bootstrapped" internally to realize the full photometric precision of which the instrument is capable, the accuracy of our calibration has increased during the past year. Thus, Table 2 contains columns for objects observed during 1988 January - March and June - July. The photometry, though still preliminary, is much better for the latter observing period - this is reflected in the increased percentage of truly blue objects identified during this period. We anticipate calibration to about $0.01 \mathrm{mag}$ at $\mathrm{mV}=18$ as the ultimate goal towards which we are working. At this level, the percentage of correctly identified candidates will again increase dramatically. Additionally, because of uncharacteristically poor luck with weather, we have yet to observe the majority of our candidates with $m_{V} \geq 18$. Despite this, we have been able to identify brighter white dwarfs, subdwarfs, quasars, etc.

In Table 2 we have included subdwarfs in the basic stellar groups. Cataclysmic and related systems are included as "composite." No spatial information other than visual inspection was used to discriminate against galaxies, hence the inclusion of a few faint galaxies with bright nuclei. The two M stars were included from CTI colors which were very red; one of these is a dMe star. 
TABLE 2: Preliminary Results

\begin{tabular}{|c|c|c|c|c|c|}
\hline \multirow{2}{*}{ Type of Object } & \multirow[t]{2}{*}{ Total } & \multicolumn{2}{|c|}{ January - March } & \multicolumn{2}{|c|}{ June - July } \\
\hline & & $\mathrm{m}_{\mathrm{v}}<17.0$ & $m_{v} \geq 17.0$ & $\mathrm{~m}_{\mathbf{y}}<17.0$ & $\mathrm{~m}_{\mathrm{v}}>17.0$ \\
\hline DA's & 8 & 1 & 4 & 0 & 3 \\
\hline Quasars & 3 & $\mathbf{0}$ & 2 & 0 & 1 \\
\hline Seyferts & 2 & 1 & 0 & $\mathbf{0}$ & 1 \\
\hline Other Galaxies & 3 & $\mathbf{0}$ & 2 & 0 & 1 \\
\hline B stars & 21 & 13 & 1 & 5 & 2 \\
\hline A stars & 21 & 14 & 1 & 4 & 2 \\
\hline F stars & 35 & 13 & 12 & 4 & 6 \\
\hline G stars & 10 & 2 & 5 & 3 & 0 \\
\hline M stars & 2 & 0 & 0 & 1 & 1 \\
\hline Composites & 4 & 3 & 0 & 1 & $\mathbf{0}$ \\
\hline Planetary Nebulae & 1 & 0 & 0 & 1 & 0 \\
\hline Totals & 110 & 47 & 27 & 19 & 17 \\
\hline
\end{tabular}

In the selection of candidates, we have utilized a technique for inspecting color $\mathrm{N}$-space by making linear combinations of color, which correspond to arbitrary projections into a plane. Figure 1 shows such a diagram utilizing the $B, V, R, I$ filter data in which blackbody colors are very nearly degenerate. This corresponds to a projection perpendicular to the main sequence. The objects we have observed thus far are marked. Now that we have confidence that our colors reliably indicate the types of objects we anticipate, we shall concentrate efforts on spectroscopically identifying the bluer objects in this diagram.

\section{Spectroscopically Identified White Dwarfs:}

Of the 110 objects thus far observed, eight are DA white dwarfs. Table 3 lists these stars along with preliminary $\mathrm{V}$ magnitude. The positions are encoded into the object name following the "CTI" designation. The positions are based on the J2000 equinox and are for epoch 1987.5. The number of characters per name is forced upon us in order to ensure uniqueness for each object in the CTI survey. CTI 104847.9+275823, also known as WD 1046+281, is the only white dwarf in the strip which has previously been spectroscopically identified (McCook and Sion 1987).

We have compared the positions of these eight white dwarfs with positions derived from the Palomar Sky Survey plates. The positions of the white dwarfs and a network of about 10 stars per object within a radius of four arcmin were measured using the two-axis Grant measuring engine at the National Optical Astronomy Observatory. Additional SAO stars across each POSS plate were also measured. Limits to the motion were derived from comparing positions on the POSS to positions derived from CTI data. POSS positions have estimated uncertainties of \pm 0.1 arcsec 
while CTI positional uncertainties are about \pm 0.2 arcsec. Within these limits, seven of the eight DA dwarfs showed no detectable motions, but the eighth, CTI 195027.4+275957, shows a motion of 0.20 arcsec $\mathrm{yr}^{-1}$. Its galactic coordinates are $l=64.64$ and $b=0.48$, with a motion vector derived over the period 1951.5 to 1987.5 of $\Delta l=\Delta b=+0.14 \operatorname{arcsec} \mathrm{yr}^{-1}$. Another object with galactic coordinates $l=+51.92$ and $b=45.36$ which is not yet spectroscopically identified, has a proper motion of 0.57 arcsec $\mathrm{yr}^{-1}$ moving principally north, perpendicular to the galactic plane. Clearly, comparing positions of CTI objects to those derived from the POSS will yield a number of interesting high motion objects. We plan to accomplish this comparison by simply digitizing the CTI strip on the POSS and utilizing the CTI software pipeline to accomplish the astrometry.

\section{TABLE 3: Spectroscopically Identified White Dwarfs}

\begin{tabular}{llll} 
Object & $\mathrm{m}_{\mathbf{v}}$ & Date Observed & Telescope \\
\hline CTI 035037.2+280044 & 18.0 & 15 Jan 1988 & MMT \\
CTI 045934.1+280335 & 17.2 & 15 Jan 1988 & MMT \\
CTI 054438.5+280224 & 17.9 & 15 Jan 1988 & MMT \\
CTI 104847.9+275823 & 15.6 & 15 Jan 1988 & MMT \\
CTI 135700.6+280448 & 17.1 & 13 Mar 1988 & $2.3 \mathrm{~m}$ \\
CTI 143808.4+275934 & 17.9 & 22 Jun 1988 & $2.3 \mathrm{~m}$ \\
CTI 163440.3+280306 & 17.1 & 11 Jul 1988 & $2.3 \mathrm{~m}$ \\
CTI 195027.4+275957 & 17.9 & 11 Jul 1988 & $\mathbf{2 . 3 ~ m}$ \\
\hline
\end{tabular}

\section{Conclusions:}

The CTI survey will be a valuable resource for discovering statistically complete samples of white dwarf (and other) stars utilizing an accurate, homogeneous photometric data set spanning colors from the ultraviolet to the red. The statistics of a complete sample grow in importance as we attempt to define the number of evolutionary "channels" capable of producing white dwarfs (e.g., Shipman 1987, Fontaine and Wesemael 1987). We have thus far done spectroscopic observations intended to explore the validity of our colors, which are still undergoing calibration improvements. Our selection of white dwarf candidates will improve as this calibration progresses.

We have discovered seven new spectroscopically identified white dwarfs and this number will increase with available observing time. These objects will be made known to the community as they are discovered.

The positions derived from CTI data are of sufficient precision to discover high motion objects when compared to POSS-derived positions. The CTI pipeline is capable of directly discovering motion objects by comparing digitized POSS data to the CTI data bases.

\section{Acknowledgements:}

We gratefully acknowledge the contributions of Michael Cawson, Tom Hess, Charles Bridges and Brian Schmidt to this effort. We thank NOAO for access to the Grant machine. The CTI project is funded by NSF and NASA and is supported by the Data General Corporation. 


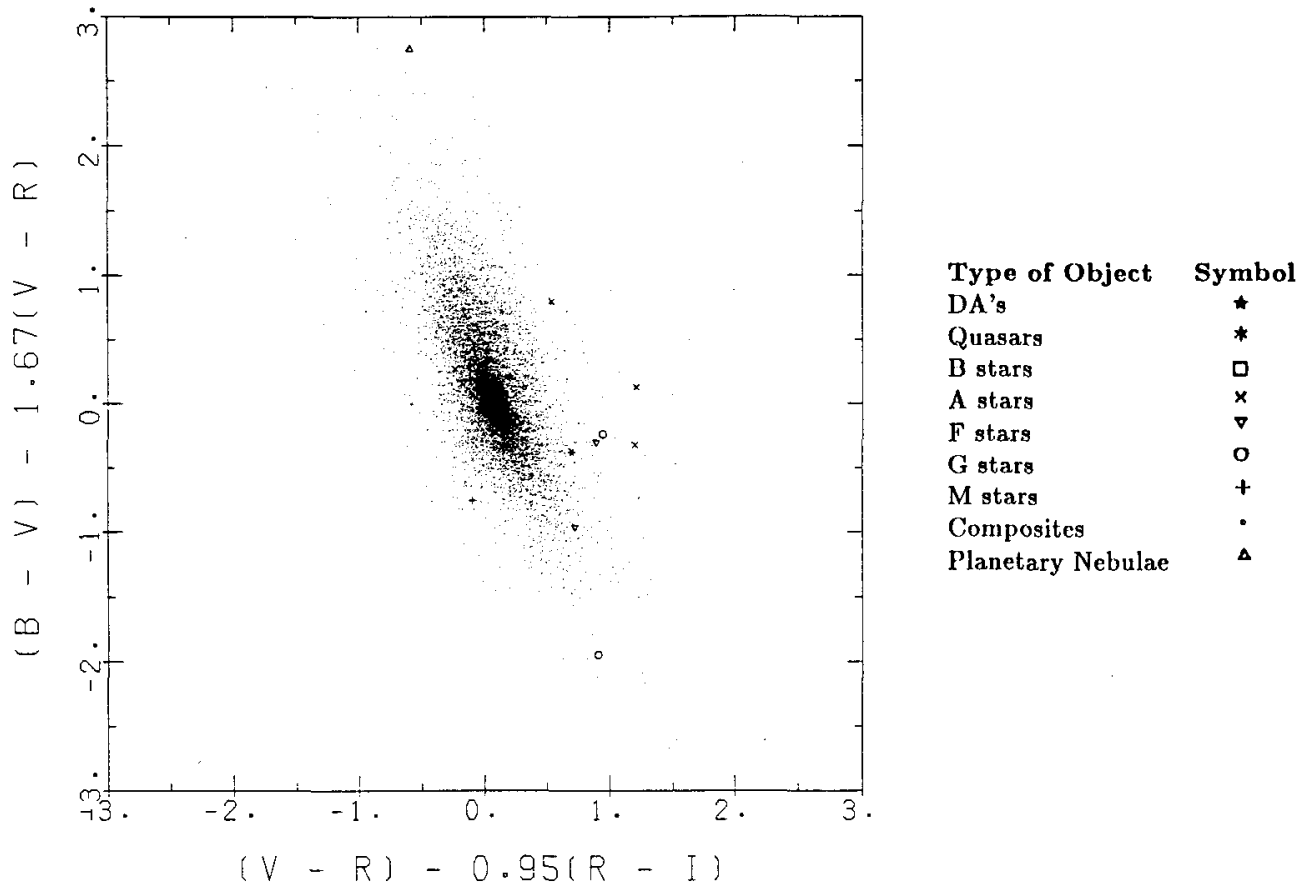

Figure 1: A special plot whose axes are linear color combinations chosen because they are nearly blackbody degenerate; the main sequence stars occupy a minimal area of the plot. This figure shows some 33000 objects, 28 of which are objects spectroscopically identified. The symbols are given in the legend above. It should be noted that there are a fair number of obvious outliers which have not yet been spectroscopically identified. There are three possible reasons why any one of these has not yet been observed: (1) The object has colors which are unreliable because of a crowded field, charge "bleeding", etc. (i.e., this plot contains all of the CTI images, not just the good ones). (2) The object is at some right ascension which has so far been unobservable. (3) The object has a magnitude which is fainter than about 18.00 - we just have not had sufficient time on the MMT to adequately observe many of the fainter candidates yet. This last reason is by far the most probable explanation for the number of unidentified outliers.

\section{References:}

Fontaine, G. and Wesemael, F. 1987, The Second Conference on Faint Blue Stars, Proc. IAU Colloq. 95, eds. A. G. D. Philip, D. S. Hayes and J. W. Liebert, 319.

McCook, George P. and Sion, Edward M. 1987, Ap. J. Suppl., 65, 603.

McGraw, J. T., Cawson, M. G. M. and Keane, M. J., Instrumentation in Astronomy VI, D. L. Crawford, Editor, Proc. SPIE 627, 60.

Shipman, H. L. 1987, The Second Conference on Faint Blue Stars, Proc. IAU Colloq. 95, eds. A. G. D. Philip, D. S. Hayes and J. W. Liebert, 273. 